\title{
Recognizing Facial Expression Using Particle Filter Based Feature Points Tracker
}

\author{
Rakesh Tripathi and R. Aravind \\ Department of Electrical Engineering, Indian Institute of Technology Madras, \\ Chennai-36, India
}

\begin{abstract}
The paper focuses on an evaluation of particle filter based facial feature tracker. Particle filter is a successful tool in the non-linear and the non-Gaussian estimation problems. We developed a particle filter based facial points tracker with a simple observation model based on sum-of-squared differences (SSD) between the intensities. Multistate face component model is used to estimate the occluded feature points. The important distances are calculated from tracked points. Two kinds of classification schemes are considered, the hidden Markov model (HMM) as sequence based recognizer and support vector machine (SVM) as frame based recognizer. A comparative study is shown in the classification of five basic expressions, i.e., anger, sadness, happiness, surprise and disgust. The tests are conducted on Cohn-Kanade and MMI face expression databases.
\end{abstract}

\section{Introduction}

Facial expression is an important cue for humans communication and emotion recognition. Expression recognition [1, 2, [3, [5] provides computers an aid to understanding human behavior. Facial expression is a necessary feedback from the user. It has many applications in behavioral science, medicine, security, manmachine interaction and animation. There are three basic elements of a facial expression recognition system, i.e., feature extraction, data representation and classification. Feature extraction involves the capture of deformation in face during the change of expression. So we need to track the important (key) points with precision. The recognition rate is affected by the goodness of tracked face points. A variety of methods exist for tracking.However, accuracy in the estimation of displacement is always under scrutiny. Here, we present a particle filter [6, [7] based feature tracker for the key points. Motivated from [8] work, the SSD based observation likelihood is formulated. At this stage, we assumed that the key-points are given for expression recognition in first frame. For our implementation, we have manually marked the facial points in the first frame. Our first goal is to track key-points in a face video which must be robust to large deformable motion, low textured region, shadows, small illumination variation (specially eye region with spectacles), occlusion due to some expressions and facial hair. The particle filter based tracker is robust to all above situations except occlusion due to some expressions. The geometric information from face

A. Ghosh, R.K. De, and S.K. Pal (Eds.): PReMI 2007, LNCS 4815, pp. 58455912007.

(C) Springer-Verlag Berlin Heidelberg 2007 
[3] is used to recover the occluded points. The euclidean distances between face features are computed using these tracked points.

It is shown in [4] that the tracking of feature points are very effective in producing facial action coding system (FACS). Most of the efforts made to know FACS using points tracking [3], 4, 7], and it is believed that the accuracy of FACS detection directly related to facial expression. Though, we are interested to produced rigorous results for expression recognition without using any rule file created from FACS. HMM [1] and SVM [5] are used for evaluation of features obtained from optical flow computation. Motivated from these works, we formed our second research goal as the evaluation of the goodness of tracked points in different classification schemes. The performance of extracted features is evaluated using both the classifiers, i.e., sequence based classifier the HMM, and frame based classifier the SVM. The results are shown on Cohn-Kanade and MMI face expression database [9], 10], and a set of videos (with 8 subjects) captured in our laboratory.

\section{Particle Filter Based Facial Points Tracker}

The particle filter employs a set of weighted particles $\left\{\mathbf{x}_{k}^{i} ; i=1, \ldots, N_{s}\right\}$ at time $k$ with associated weights $\left\{w_{k}^{i} ; i=1, \ldots, N_{s}\right\}$ to represent the posterior pdf $p\left(\mathbf{x}_{k} \mid \mathbf{z}_{1: k}\right)$. The weights are normalized such that $\sum_{i} w_{k}^{i}=1$. If $\left\{\mathbf{z}_{1: k}^{i} ; i=\right.$ $\left.1, \ldots, N_{s}\right\}$ is a set of all available measurements up to time $k$, then the posterior density at $k$ can approximated as $p\left(\mathbf{x}_{k} \mid \mathbf{z}_{1: k}\right) \approx \sum_{i=1}^{N_{s}} w_{k}^{i} \delta\left(\mathbf{x}_{k}-\mathbf{x}_{k}^{i}\right)$. The sequential importance sampling/resampling particle filter for our application is described below.

1. State Prediction: The state is defined to be the coordinate of particle location. Let the location of $i$ th particle of $k$ th frame corresponding to a feature point is denoted by $\left(x_{k}^{i}, y_{k}^{i}\right)$ and intensity at this point by $I_{k}\left(x_{k}^{i}, y_{k}^{i}\right)$, so that the state is $\mathbf{x}_{k}^{i}=\left[\begin{array}{ll}x_{k}^{i} & y_{k}^{i}\end{array}\right]$. The transition model is linear with the Gaussian noise of zero mean and known variance $\sigma^{2}$ as process noise $\mathbf{v}_{k}$. The transition equation is $\mathbf{x}_{k}^{i}=\mathbf{x}_{k-1}^{i}+\mathbf{v}_{k}$.

2. Formulation of the Observation Model: Our observation model is based upon the sum-of-squared-differences (SSD) described in 8]. We define the observation $\mathbf{z}_{k}$ at a given $\mathbf{x}_{k}^{i}$ to be the image intensities at pixel locations in the window of size $(2 N+1) \times(2 N+1)$. The displacement between $i_{t h}$ particle of a feature point in the first frame located at $\left(x_{1}^{i}, y_{1}^{i}\right)$, and in the $k_{t h}$ frame at $\left(x_{k}^{i}, y_{k}^{i}\right)$ is $\left.\left(d_{x}^{i}, d_{y}^{i}\right)=\left(x_{1}^{i}-x_{k}^{i}, y_{1}^{i}-y_{k}^{i}\right)\right)$. The SSD is defined as,

$D\left(d_{x}^{i}, d_{y}^{i}\right)=\sum_{m=-N}^{N} \sum_{n=-N}^{N}\left(\frac{I_{1}\left(x_{1}^{i}+m, y_{1}^{i}+n\right)}{M_{1}}-\frac{I_{k}\left(x_{1}^{i}+d_{x}^{i}+m, y_{1}^{i}+d_{y}^{i}+n\right)}{M_{k}^{i}}\right)^{2}$ 
where $M_{1}$ is mean of image intensity in the window of marked point by user in the first frame, and $M_{k}^{i}$ is mean of intensity in the window of frame $k$ and particle $i$.

3. Weighting the Samples: The response distribution $R\left(d_{x}^{i}, d_{y}^{i}\right)$ is formulated as, $R\left(d_{x}^{i}, d_{y}^{i}\right)=\exp \left(-D\left(d_{x}^{i}, d_{y}^{i}\right) / \sigma_{s}^{2}\right)$, where $\sigma_{s}$ is a scaling parameter given as $\sigma_{s}=\tau / M_{1}$. The normalized weight $w_{k}^{i}=R\left(d_{x}^{i}, d_{y}^{i}\right) / \sum_{i} R\left(d_{x}^{i}, d_{y}^{i}\right)$.

4. State Update and Estimation: The resampling eliminates particles that have small weights and concentrate on particles with large weights. In our case, SSD is very low at positions of exact match and hence likelihood is very high. Hence, we need to do resampling at each time step [6]. The posterior mean estimator is used for determining the position of feature points. The resampling algorithm produces equally weighted particles, so the posterior mean estimate $\mathbf{S}_{k}^{\text {Mean }}$ at time step $k$ is given by

$$
\mathbf{S}_{k}^{\text {Mean }}=\sum_{i=1}^{N_{s}} w_{k}^{i} \mathbf{x}_{k}^{i}=\frac{1}{N_{s}} \sum_{i=1}^{N_{s}} \mathbf{x}_{k}^{i}
$$

\subsection{Incorporating Face Geometry}

The results of tracking using particle filter is shown in Fig. 1] It works well for expressions having enough observations for each points. However, for the tightly closed lip and the closed eye as in anger (see third figure of Fig. 11), particle filter cannot track the exact position of center of upper and lower lip and eyelid. It is because of the SSD based likelihood, partial occlusion of key points make the likelihood very low at true positions. However, if we can know that the mouth (or eye) is tightly closed, then the position of center points of upper and lower lip (or eyelid) is the middle point of two corners of mouth (or eye). To know the state of features (tightly closed or open), we used a variant of multistate facial component model (MFCM) proposed in [3. A method to detect the state of mouth and eye is described in 3. The number of lip pixels in tightly closed mouth is very less than open mouth situation. The lip and non-lip pixels are modeled and classified by the Gaussian mixture model. Whereas, the presence
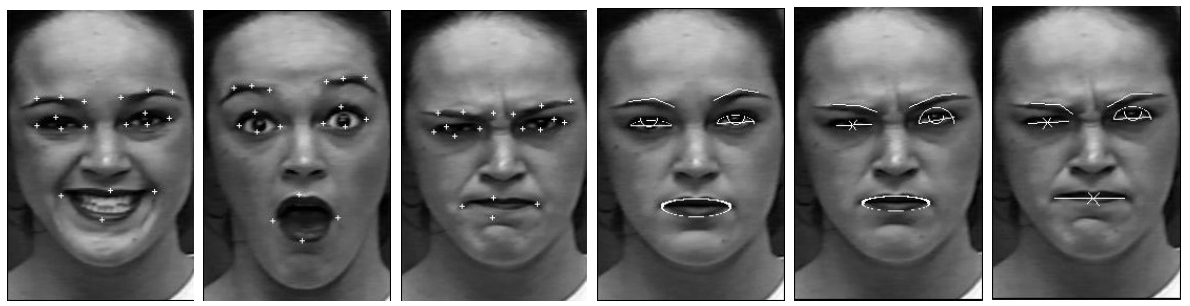

Fig. 1. Tracking using particle filter and MFCM representation 
of iris gives important information regarding state of eye. The iris is visible for open eye only. The edginess image [1] followed by binary conversion gives good discriminating information for the presence of iris (Fig. 2(a) and Fig. 2(c)]. The iris mask shown in Fig. 2(b) is used to calculate the threshold based on number of white pixels in the region of mask and average iris intensity [3]. The change of states is shown in last two figures of Fig. 1.
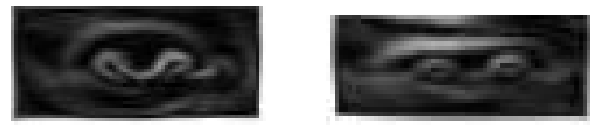

(a) Edginess image for open and closed eyes.
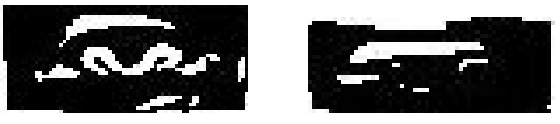

(c) Corresponding binarized images.

Fig. 2. Iris is searched between left and right eye corners with mask shown in Fig. 2(b)

\section{Facial Expression Recognition}

\subsection{Feature Representation}

We calculated the Euclidean distances between tracked feature points. These distances represent the change of facial expression [3], 7]. Upper face consists the motion of eye, brow, cheek and forehead, and gives 9 parameters and lower face consists motion of mouth, and gives 8 parameters listed in Table 1 and shown in Fig. 3 .

\subsection{Classification of Expressions}

Two classifiers are used to evaluate the goodness of extracted features, sequence based and frame based. The sequence based recognizer exploits the temporal relationship between adjacent frames along with the spatial information. The HMM is widely used for making a sequence of decisions from an interconnected observations. Five HMMs are modeled using Baum-Welch algorithm, one for each expression: anger, sadness, happiness, disgust and surprise. The log-likelihood obtained using forward algorithm from each HMM is used to classify an expression corresponding to the highest probability. HMM delays the decisions by 10 
Table 1. Important distances. Subscript 0 represents first frame distance.

\begin{tabular}{|c|c||c|c|}
\hline Inner brow & $\frac{H L 2-H L 2_{0}}{H L 2_{0}}$ & Lip height & $\frac{(H U+H L)-\left(H U_{0}+H L_{0}\right)}{\left.H U_{0}+H l_{0}\right)}$ \\
\hline Outer brow & $\frac{H L 1-H L 1_{0}}{H L 1_{0}}$ & Lip Width & $\frac{W-W_{0}}{W}$ \\
\hline Eye Height & $\frac{(H E L 1+H E L 2)-\left(H E L 1_{0}+H E L 2_{0}\right)}{H E L 1_{0}+H E L 2_{0}}$ & Lip corner & $\frac{H L C-H L C_{0}}{H L C C_{0}}$ \\
\hline Brows distance & $\frac{D-D D_{0}}{D_{0}}$ & Top lip & $\frac{H U-H U_{0}}{H U_{0}}$ \\
\hline Cheek motion & $\frac{C L C L_{0}}{C L}$ & Bottom lip & $\frac{H L-H L_{0}}{H L L_{0}}$ \\
\hline $\begin{array}{c}\text { Nose to upper } \\
\text { lip width }\end{array}$ & $\frac{H N N_{0}}{H N_{0}}$ & Chin motion & $\frac{H C-H C_{0}}{H C_{0}}$ \\
\hline
\end{tabular}

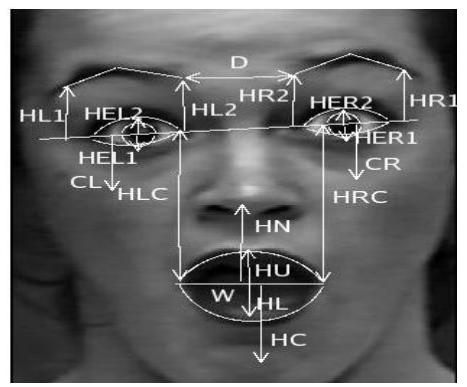

Fig. 3. Important distances between face features

to 15 frame because first it generates all the connected hidden sequence of certain length and then calculates the likelihood for the decisions based on optimal sequence of hidden states.

The frame based recognizer gives decision after each frame independent of previous decisions. Although the frame based recognizer loose the temporal information between adjacent frames, recognition at frame level is specially useful in the situations of mixture of expressions in a single video. We used SVM with Gaussian kernel as frame level classifier. Features calculated from each frame are separately fed to SVM for training and classification. The one-against-all strategy is applied in multiclass classification with SVM.

\section{Experimental Results}

The database is re-sized to $180 \times 280$ from cropped facial image using bicubical interpolation. All the frames have frontal view of the face with small in-plane rigid head motion. It is assumed that key-points have limited motion of 20 to 40 pixels. Total 21 facial points are tracked. The SSD window size is $13 \times 13$. Fifteen important frames per video is chosen, showing the change of expression from the neutral.

For the classification using HMM, each frame is represented by a vector of 17 Euclidean distances, and each video is converted into temporally connected 
sequence (observable). The statistics regarding the number of training and testing subjects with different kinds of database in shown in Table 2 and Table 3 The subjects used for training is not included to determine the recognition rates (person independent test). The average recognition rates are calculated by comparing human observed correct number of videos classified in rest of the videos. We also captured the expression videos of 8 persons in our laboratory with a motivation of measuring performance with different kind of training sets, keeping the same parameters for feature extraction (different thresholds) Table 4. However, sad expression is felt difficult to produce, so results are shown only with 4 expressions.

It is observed that the features associated with surprise and happiness expressions are much discriminative than other expressions. We can recognize these two expressions from all the videos of Cohn-Kanade database correctly using the lower face features only. Similarly in case of MMI database except one video, all other videos of these two expressions are recognized correctly. There is no ambiguity in the recognition of surprise and happiness expressions using full face features. It is seen that the lower face features for disgust expression is not discriminative. However, the more videos can be recognized by using full face features. Anger and sadness expressions have mutually overlapped features. We felt that the sadness is most difficult expression to recognize. The recognition performance of various training sets with our made database is shown in Table 4. It can be observed that the parameters used for different database are tuned good in comparison to the results from different database.

Table 2. Classification with 3 training and 7 test videos of Cohn-Kanade face expression database using HMM

\begin{tabular}{|c|c|c|c|c|c|c|}
\hline Face Features & Surprise & Sadness & Anger & Disgust & Happiness & $\begin{array}{c}\text { Avg. recognition } \\
\text { rates (in \%) }\end{array}$ \\
\hline Lower & 7 & 4 & 4 & 3 & 7 & 71.5 \\
\hline Upper+Lower & 7 & 4 & 5 & 5 & 7 & 80 \\
\hline
\end{tabular}

Table 3. Classification with 3 training and 9 test videos of MMI face expression database using HMM

\begin{tabular}{|c|c|c|c|c|c|c|}
\hline Face Features & Surprise & Sadness & Anger & Disgust & Happiness & $\begin{array}{c}\text { Avg. recognition } \\
\text { rates (in \%) }\end{array}$ \\
\hline Lower & 8 & 4 & 5 & 5 & 8 & 66.7 \\
\hline Upper+Lower & 9 & 6 & 6 & 7 & 9 & 82.2 \\
\hline
\end{tabular}

An extra class of neutral face action is considered while classification using SVM because all the videos have first few frames as neutral. The SVM is trained with 120 frames (100 with expression and 20 neutral). The experimental results with different kinds of database is given in Table 5 and Table 6. It should be noted 
Table 4. Classification with videos captured in our lab. of 8 persons using HMM

\begin{tabular}{|c|c|c|c|c|c|c|}
\hline Training set & $\begin{array}{c}\text { Face } \\
\text { features }\end{array}$ & Surprise & Anger & Disgust & Happiness & $\begin{array}{c}\text { Average } \\
\text { recog. rate }\end{array}$ \\
\hline \multirow{2}{*}{ Cohn-Kanade } & Lower face & 8 & 5 & 4 & 7 & 75 \\
\cline { 2 - 7 } & Full face & 8 & 7 & 5 & 7 & 84.4 \\
\hline \hline \multirow{2}{*}{ MMI } & Lower face & 7 & 4 & 4 & 7 & 68.8 \\
\cline { 2 - 7 } & Full face & 8 & 5 & 5 & 7 & 78.1 \\
\hline
\end{tabular}

that these recognition rates are obtained from the confusion matrix using oneagainst-all strategy. The recognition rate is calculated by observing the correct number of frames within the total number of frames of same expression and given in percentage. The number of samples used for testing are 60 frames for each class with the Cohn-Kanade database and 70 frames with the MMI database. The neutral expression is not considered in determining average recognition rate from SVM because the number of samples for neutral is very high (200 frames in Cohn-Kanade database and 280 frames in MMI database), first few frames of each video, and recognition rate for neutral expression is more than $94 \%$. The major difference in the classification by both classifier in found in the case of disgust and anger. The SVM performs better than HMM for recognizing disgust using only lower face features. Whereas the results from anger is just opposite to that of disgust.

Table 5. Recognition rates (in \%) with 120 training and 500 test frames (200 neutral and 60 per expression) of Cohn-Kanade face expression database using SVM

\begin{tabular}{|c|c|c|c|c|c|c|c|}
\hline $\begin{array}{c}\text { Face } \\
\text { Features }\end{array}$ & Neutral & Surprise & Sadness & Anger & Disgust & Happiness & $\begin{array}{c}\text { Avg. recognition } \\
\text { rates }\end{array}$ \\
\hline Lower & 94.6 & 93.6 & 76.0 & 36.0 & 77.5 & 84.2 & 73.5 \\
\hline Upper+Lower & 96.6 & 98.2 & 84.1 & 50.1 & 78.7 & 79.0 & 78.0 \\
\hline
\end{tabular}

Table 6. Recognition rates (in \%) with 120 training and 630 test frames (280 neutral and 70 per expression) of MMI face expression database using SVM

\begin{tabular}{|c|c|c|c|c|c|c|c|}
\hline $\begin{array}{c}\text { Face } \\
\text { Features }\end{array}$ & Neutral & Surprise & Sadness & Anger & Disgust & Happiness & $\begin{array}{c}\text { Avg. recognition } \\
\text { rates }\end{array}$ \\
\hline Lower & 91.4 & 89.4 & 50.2 & 51.3 & 71.5 & 87.4 & 70 \\
\hline Upper+Lower & 92.2 & 91.2 & 64.7 & 58.5 & 77.4 & 89.2 & 76.2 \\
\hline
\end{tabular}

\section{Conclusion}

In this paper, we evaluated the goodness of features obtained from particle filter based feature points tracker. The particle filter with the Gaussian prior and 
linear transition model is able to generate particles in interesting regions. The SSD based observation model is enough robust to give the information from low textured regions also. However, the expressions like anger occludes the feature points. So the output from the particle filter is modified using geometrical information from face. The HMM and the SVM can classify the two expressions surprise and happiness clearly. With HMM sad is the most difficult expression to recognize, whereas features associated with anger gives poor performance with SVM. In general full face features gives improvement in the classification. We feel that the classification performance can be improved for the other expressions by using transient features also [3].

\section{References}

1. Yacoob, Y., Davis, L.S.: Recognizing Human Facial Expression from Long Image Sequences using Optical Flow. IEEE Trans. PAMI 18(6), 636-642 (1996)

2. Bartlett, M.S., Braathen, B., Littlewort-Ford, G., Harshey, J., Fasel, I., Marks, T., Smith, E., Sejnowski, T., Movellen, R.L.: Automatic analysis of spontaneous facial behaviour: A final project report, Technical Report INC-MPLab, UCSD (2001)

3. Tian, Y., Kanade, T., Cohn, J.: Recognizing action unit for facial expression analysis. IEEE Trans. PAMI 23(2), 97-114 (2001)

4. Cohn, J.F., Zlochower, A.J., Lian, J., Kanade, T.: Automated face analysis by feature point tracking has high concurrent validity with manual FACS coding. Psycophysiology 36, 35-43 (1999)

5. Anderson, K., McOwan, P.W.: A real time automated system for the recognition of human facial expression. IEEE Trans. System, Man, and Cyb.-PartB: Cyb. 36(1), 96-105 (2006)

6. Arulampalam, M.S., Maskell, S., Gordan, N., Clapp, T.: A Tutorial on particle filter for online nonlinear/non-Gaussian Bayesian tracking. IEEE Trans. Signal Processing 50(2), 172-188 (2002)

7. Valstar, M.F., Patras, I., Pantic, M.: Facial action unit detection using probabilistic actively learned support vector machines on tracked facial Point data. In: CVPR 2005

8. Singh, A., Allen, P.: Image flow computation: An estimation-theoretic framework and a unified perspective. CVGIP: Image Understanding 56(2), 152-177 (1992)

9. Kanade, T., Cohn, J.F., Tian, Y.: Comprehensive database for facial expression analysis. In: Fourth International Conference on Automatic Facial and Gesture Recognition, Grenobel, France (2004)

10. Pantic, M., Valstar, M.F., Rademaker, R., Maat, L.: In: Proc. IEEE Int'l Conf. Multmedia and Expo (ICME 2005), Amsterdam, The Netherlands (July 2005)

11. Kumar, P.K., Das, S., Yegnanarayana, B.: One Dimension processing of the images. In: International Conference on Multimedia Processing Systems, Chennai, India (2000) 